\title{
Direct optical excitation of two and three magnons in $\alpha-\mathrm{Fe}_{2} \mathrm{O}_{3}$
}

\author{
Y. Tanabe ${ }^{1}$, Y. Fujimaki ${ }^{2}$, K. Kojima ${ }^{2}$, S. Uchida ${ }^{2}$, \\ S. Onari ${ }^{3}$, T. Matsuo ${ }^{3}$, S. Azuma ${ }^{4}$, and E. Hanamura ${ }^{4}$ \\ ${ }^{1}$ Department of Applied Physics, University of Tokyo, 7-3-1 Hongo, Tokyo 113-8656, Japan \\ ${ }^{2}$ Department of Physics, University of Tokyo, 7-3-1 Hongo, Tokyo 113-8656, Japan \\ ${ }^{3}$ Institute of Materials Science, University of Tsukuba, Tsukuba, Ibaraki 304-8573, Japan \\ ${ }^{4}$ Chitose Institute of Science and Technology and Japan Science and Technology Agency \\ Chitose, Hokkaido 066-8655, Japan \\ Email: hanamura@photon.chitose.ac.jp
}

Received December 20, 2004

\begin{abstract}
Direct excitation of two and three magnons is observed in midinfrared absorption and Raman scattering spectra of $\alpha-\mathrm{Fe}_{2} \mathrm{O}_{3}$ crystals. These polarization characteristics and the spectra themselves are shown to be understood from group-theoretical point of view. The microscopic mechanism of three-magnon excitation is proposed in addition to that of well-known two-magnon excitation process.
\end{abstract}

PACS: 75.30.Ds, 78.30.-j, 75.50.Ee

\section{Introduction}

Transition-metal oxide with open $d$-electron shells have shown many interesting magnetic and transport phenomena of strongly correlated $d$-electrons [1]. Optical absorption due to phonon-assisted two-magnon excitation was observed for transition-metal oxides of quasi-two--dimensional $\mathrm{La}_{2} \mathrm{CuO}_{4}, \quad \mathrm{Nd}_{2} \mathrm{CuO}_{4}, \quad \mathrm{Sr}_{2} \mathrm{CuO}_{2} \mathrm{Cl}_{2}$ [2], $\mathrm{La}_{2} \mathrm{NiO}_{4}$ [3], $\mathrm{YBa}_{2} \mathrm{Cu}_{3} \mathrm{O}_{6}$ [4], and two-leg-ladder $(\mathrm{Ca}, \mathrm{La})_{14} \mathrm{Cu}_{24} \mathrm{O}_{41}$ crystals [5]. On the other hand, phonon-assisted particle-hole excitation of the spinons was confirmed in quasi-one-dimensional $\mathrm{Sr}_{2} \mathrm{CuO}_{3}$ crystal [6]. Here the oscillator strength of the optical phonon makes the optical excitation of two magnons possible through the magnon-phonon interaction [7].

We have observed direct midinfrared absorption by two- and three-magnon excitation in $\alpha-\mathrm{Fe}_{2} \mathrm{O}_{3}$ crystal [8]. This is due to the effective electric dipole moment induced by the combined effect of the electronic transition of the $d$-electron and the exchange interaction between the neighboring $\mathrm{Fe}^{3+}$ ions $[9,10]$. The magnon energy as well as the absorption coefficient of $\alpha-\mathrm{Fe}_{2} \mathrm{O}_{3}$ are much larger than those of $\mathrm{MnF}_{2}$ with Néel tempera- ture $T_{N}=67.3 \mathrm{~K}$ and absorption coefficienct of a few $\mathrm{cm}^{-1}[11,12]$.

Magnetic properties of $\alpha-\mathrm{Fe}_{2} \mathrm{O}_{3}$ have been well studied [13] and the origin of weak ferromagnetism was proposed from the studies of this crystal [14,15]. The magnon dispersion has been obtained from inelastic neutron scattering investigation [16] and also from its theoretical analysis [17]. On the other hand, the phonon structure of $\alpha-\mathrm{Fe}_{2} \mathrm{O}_{3}$ is also obtained by the infrared reflection spectrum [18], Raman scattering [19] and lattice dynamics study [20]. Two-magnon Raman scattering in this crystal had been a controversial point but this was also clarified by the effects of pressure and isotope substitution on the Raman spectrum [21-23]. Magnetic structure of this crystal was found to change at Morin temperature $T_{M}=261 \mathrm{~K}$ [24]. Below $T_{M}$, the four sublattice magnetizations are parallel to the three-fold $c$-axis of the corundum structure with the space group $D_{3 d}^{6}\left(\overline{3} \frac{2}{m}\right)$, and this phase shows pure antiferromagnetism. Between $T_{M}$ and Néel temperature $T_{N}=960 \mathrm{~K}$, the four sublattice magnetizations are almost in the $a b$-plane and the weak ferro- 
magnetism is induced in the direction of two-fold $a$-axis. Here the space group is monoclinic $C_{2 h}\left(\frac{2}{m}\right)$.

In the present paper, we shall supplement the previous report on the midinfrared absorption due to magnons [8] by supplying the observed polarizationand temperature-dependence of the Raman spectrum. Then we can confirm the assignment of two-magnon excitation at $D$-point at the Brillouin zone edge by the effective (exchange) dipole moment $\pi \mathbf{S}_{i} \cdot \mathbf{S}_{j}$. This will be given in Sec. 2. The three-magnon excitation was assigned to originate from the effective dipole moment, like in $\pi^{D M} \cdot\left(\mathbf{S}_{i} \times \mathbf{S}_{j}\right)$ [10]. We will give all the terms that contribute to the three-magnon excitation in Sec. 3 and discuss its microscopic mechanism in Sec. 4. The last Sec. 5 is for conclusion and discussion.

\section{Midinfrared and Raman scattering spectrum}

The used samples have been prepared from single crystals of good-quality natural hematite which were used also in Ref. 8. These crystals have been sliced and polished by using diamond powder and finally by aluminate powder with a diameter $0.1 \mu \mathrm{m}$. The used samples have thickness ranging from $50 \mu \mathrm{m}$ to $140 \mu \mathrm{m}$.

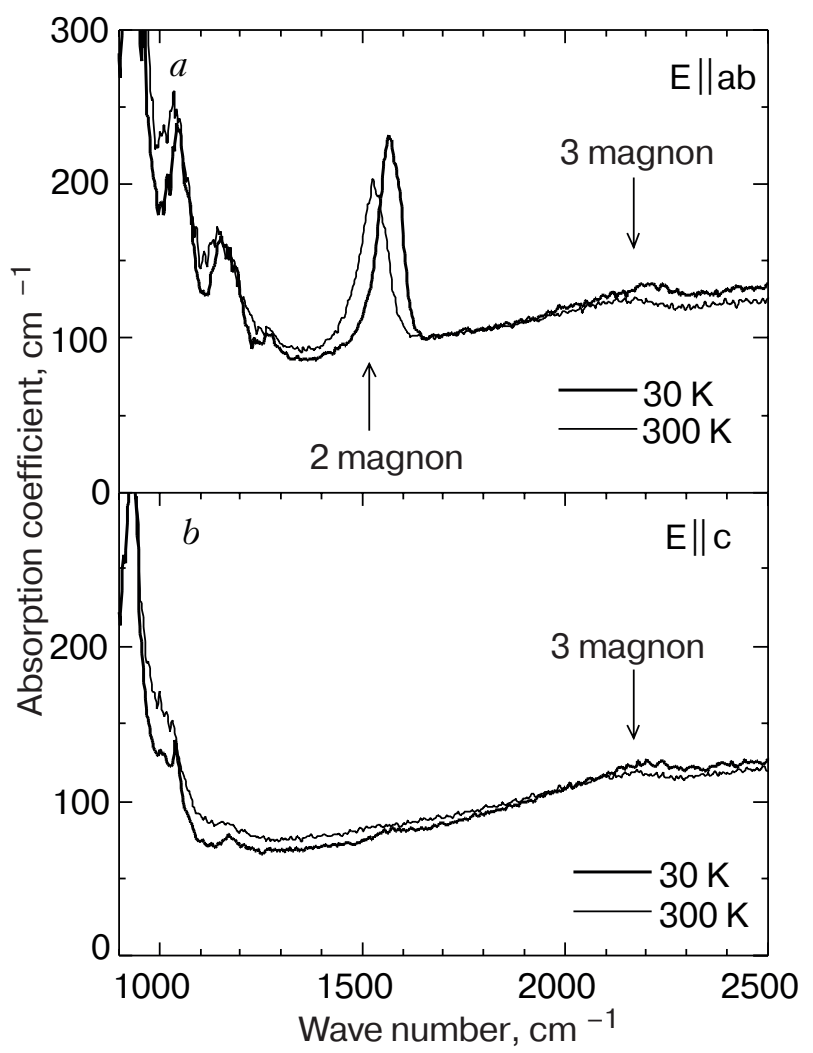

Fig. 1. The polarization dependence of midinfrared absorption spectrum of $\alpha-\mathrm{Fe}_{2} \mathrm{O}_{3}$ crystal with a crystal thickness $120 \mu \mathrm{m}$ at $30 \mathrm{~K}$ and $300 \mathrm{~K}(a)$. The incident beam is polarized in $a b$-plane and in $c$-direction $(b)$.

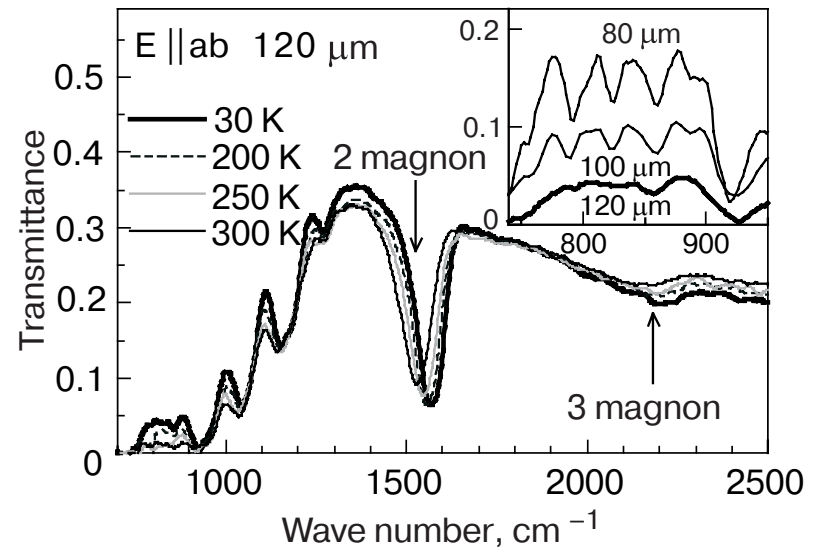

Fig. 2. The temperature dependence of the transmission spectrum for the polarization $E$ in the $a b$-plane for the same sample-thickness $120 \mu \mathrm{m}$ as in Fig. 1. The inset shows the sample-thickness dependence around $800 \mathrm{~cm}^{-1}$ at $30 \mathrm{~K}$. A single $E_{u}$ magnon is expected at $782 \mathrm{~cm}^{-1}$ while combination modes of two phonons are at $780 \mathrm{~cm}^{-1}\left(\mathrm{TO}_{2}+\mathrm{LO}_{3}\right)$, $805 \mathrm{~cm}^{-1}\left(\mathrm{TO}_{3}+\mathrm{LO}_{2}\right)$ and $851 \mathrm{~cm}^{-1}\left(\mathrm{TO}_{3}+\mathrm{LO}_{1}^{\prime}\right)$.

The measurement of the midinfrared absorption spectrum was performed with a fourier-transform spectrometer Bruker IFS $113 \mathrm{~V}$ in the energy range 350 to $2400 \mathrm{~cm}^{-1}$. We have observed several prominent features on the high energy side of the single phonon modes [18] as shown in Figs. 1 and 2. The first important characteristics is that the absorption peak just above $1500 \mathrm{~cm}^{-1}$ shows strong polarization dependence: no signal is observed for $\mathbf{E} \| c$-axis while the strong signal for $\mathbf{E} \perp c$-axis. On the other hand, the signal around $2200 \mathrm{~cm}^{-1}$ is observed under both polarizations. Second, both of these peaks show blue shift with decreasing lattice temperatures, e.g., the absorption peak of $1500 \mathrm{~cm}^{-1}$ at room temperature shifts to $1570 \mathrm{~cm}^{-1}$ at $30 \mathrm{~K}$. Third, three absorption peaks are observed on the high energy side of the single phonon modes [18], i.e., $1160 \mathrm{~cm}^{-1}, 1050 \mathrm{~cm}^{-1}$, and $920 \mathrm{~cm}^{-1}$. These energies are almost independent of the lattice temperature in contrast to those at $1500^{-1}$ and 2200 $\mathrm{cm}^{-1}$. From these polarization and temperature dependence, we may speculate that the signals at 1500 $\mathrm{cm}^{-1}$ and $2200 \mathrm{~cm}^{-1}$ come from the elementary excitations different from others.

As a matter of fact, basing upon the assignment of single phonon modes [18] observed by the infrared reflection spectrum and the magnon dispersion obtained from neutron inelastic scattering, we can assign 1160 $\mathrm{cm}^{-1}$ signal to combination of $\mathrm{TO}_{4}\left(524 \mathrm{~cm}^{-1}\right)+\mathrm{LO}_{4}$ (or $\left.\mathrm{LO}_{2}^{\prime}\right)\left(662 \mathrm{~cm}^{-1}\right), 1050 \mathrm{~cm}^{-1}$ signal to that of $\mathrm{TO}_{4}\left(524 \mathrm{~cm}^{-1}\right)+\mathrm{TO}_{2}^{\prime}\left(526 \mathrm{~cm}^{-1}\right)$, and $920 \mathrm{~cm}^{-1}$ signal to that of $\mathrm{TO}_{3}\left(437 \mathrm{~cm}^{-1}\right)+\mathrm{TO}_{2}^{\prime}\left(494 \mathrm{~cm}^{-1}\right)$. Each of the transverse and longitudinal $E_{u}$ modes is numbered from the lower to higher energy side, and 


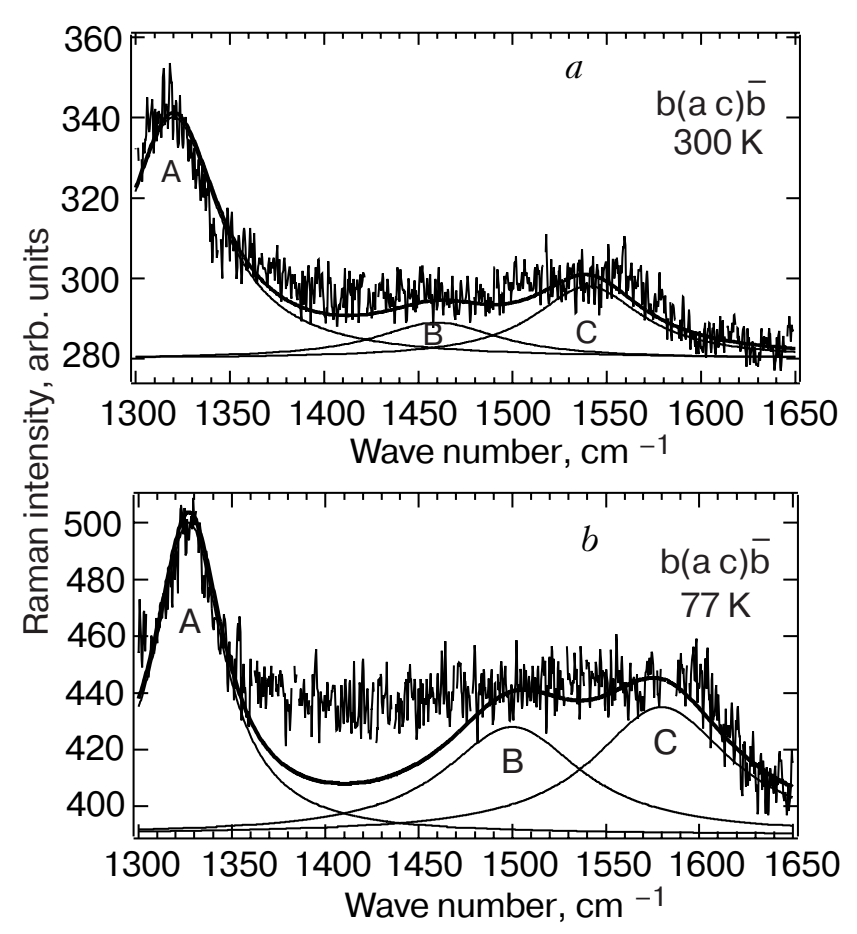

Fig. 3. Raman spectrum of $\alpha-\mathrm{Fe}_{2} \mathrm{O}_{3}$ at room temperature (a) and $77 \mathrm{~K}(b)$ with incident beam $633 \mathrm{~nm}$ from $\mathrm{He}^{-} \mathrm{Ne}$ laser. $A, B$ and $C$ describe Lorentzian fittings with (central wave number, width at half-maximum, relative intensity) = $=\left(1320 \mathrm{~cm}^{-1}, 60 \mathrm{~cm}^{-1}, 60\right)$ for $A,\left(1460 \mathrm{~cm}^{-1}, 80 \mathrm{~cm}^{-1}, 9\right)$ for $B$ and $\left(1540 \mathrm{~cm}^{-1}, 70 \mathrm{~cm}^{-1}, 18\right)$ for $C$ in $(a)$, and $(1327$ $\left.\mathrm{cm}^{-1}, 45 \mathrm{~cm}^{-1}, 110\right)$ for $A,\left(1500 \mathrm{~cm}^{-1}, 90 \mathrm{~cm}^{-1}, 38\right)$ for $B$ and $\left(1580 \mathrm{~cm}^{-1}, 90 \mathrm{~cm}^{-1}, 45\right)$ for $C$ in $(b)$. The thick solid line describes superposition of Lorentzian curves $A, B$ and $C$.

$\mathrm{LO}_{2}^{\prime}$ and $\mathrm{TO}_{2}^{\prime}$ correspond to the $A_{2 u}$ modes. The energies of $1500 \mathrm{~cm}^{-1}$ and $2200 \mathrm{~cm}^{-1}$ are nearly equal to two and three magnons, respectively, obtained from the neutron data [17]. We are interested in the wave number region around $800 \mathrm{~cm}^{-1}$ to check whether a single magnon is observed in the absorption spectrum. The transmission spectrum is observed as shown in the inset of Fig. 2 by reducing the sample thickness from $120 \mu \mathrm{m}$ to 100 and $80 \mu \mathrm{m}$.

In order to confirm these assignments, Raman spectrum has been observed with Stokes shift in this energy range. This gives information to supplement the midinfrared absorption spectrum. Figures $3, a, b$ give the Raman spectrum observed by using $\mathrm{He}-\mathrm{Ne}$ laser as incident beam at room temperature and nitrogen temperature, respectively. A doublet structure is observed with Stokes-shift $1460 \mathrm{~cm}^{-1}$ and $1540 \mathrm{~cm}^{-1}$ at room temperature as shown in Fig. 3, $a$ and shows blue-shift similar to that in the midinfrared absorption spectrum at nitrogen temperature as shown in Fig. 3,b. The stronger signal at $1320 \mathrm{~cm}^{-1}$ is almost independent of the lattice temperature and is well assigned to overtone of two $\mathrm{LO}_{4}$ phonons $\left(2 \times 662 \mathrm{~cm}^{-1}\right)$. These two-phonon excitations at 1160, 1050 and $920 \mathrm{~cm}^{-1}$ and at $1320 \mathrm{~cm}^{-1}$ obey Loudon's selection rule of combination and overtone modes, respectively, for the infrared and Raman scattering process [25]. The doublet structure around $1500 \mathrm{~cm}^{-1}$ is observed under the configuration $b(a, c) \bar{b}$ but not for $b(a, a) \bar{b}$ nor for $b(c, c) \bar{b}$ while the signal at $1320 \mathrm{~cm}^{-1}$ is allowed for both configurations. This means that the elementary excitation with doublet structure around $1500 \mathrm{~cm}^{-1}$ has the symmetry $E_{g}$.

The second task of the present paper is to understand two- and three-magnon excitations both in the midinfrared and Raman spectrum from both group-theoretical and microscopic points of view. These will be discussed in the following sections.

\section{Selection rules}

The midinfrared absorption peak due to two-magnon excitation is observed more strongly than a single magnon absorption. This is expected simply from two reasons: (i) the joint density of states for a pair of excitation $\mathbf{k}$ and $-\mathbf{k}$ which diverges at the high-symmetry points on the Brillouin zone (BZ) edges becomes much larger than that of single elementary excitation near $\Gamma$-point; (ii) and the two-magnon excitation in the antiferromagnet is accompanied by rather large electronic dipole moment due to the mechanism different from a single magnon excitation as discussed in Ref. 9. The total symmetry of the two excitons (magnons) at the high symmetry points of the $\mathrm{BZ}$ edge may be determined as given in the literature [26,27].

The magnetic unit cell of $\alpha-\mathrm{Fe}_{2} \mathrm{O}_{3}$ consists of four sublattices both below and above $T_{M}$. The effective dipole moment to excite two magnons is associated with the antiferromagnetic pair of the neighboring magnetic ions coupled by the superexchange interaction [9].

Loudon $[26,27]$ has shown how to derive the selection rules for the two-magnon absorption. The procedure is to reduce the representation at $\Gamma$-point whose bases are two-magnon states at the high symmetry point of the BZ boundary and to see whether the decomposition contains the representation the dipole moment operator belongs to. If it does, the excitation of two magnons at the boundary will be allowed, intensified there by the high density of state.

This conventional procedure can be applied to optical excitations of two magnons at high symmetry points in the Brillouin edge for antiferromagnets with two magnetic sublattices such as in $\mathrm{MnF}_{2}$. However, in the case of $\alpha-\mathrm{Fe}_{2} \mathrm{O}_{3}$ where the magnetic unit cell contains four sublattices, we must exclude the processes of two-magnon excitation from the pair with ferromagnetic coupling. As a result, the selection rule is modified as shown in Ref. 10. The modified selec- 
tion rule for direct optical excitation of two magnons in $\alpha-\mathrm{Fe}_{2} \mathrm{O}_{3}$ is summarized as follows. Symmetry put in a double bracket on the right-hand side is forbidden, either because the final state is antisymmetric or because it corresponds to the creation of two magnons both within the up- or down-sublattices. In the antisymmetric combination denoted by \{\} , two contributions cancel out each other.

(a) $Z$-point with acconstic $Z_{2}$ and optical $Z_{3}$ magnons:

$$
\left[Z_{2}^{2}\right]=\left[Z_{3}^{2}\right]=A_{1 u}+\left[\left[E_{g}\right]\right], \quad\left\{Z_{2}^{2}\right\}=\left\{Z_{3}^{2}\right\}=\left[\left[A_{2 u}\right]\right],
$$

$$
Z_{2} \times Z_{3}=A_{1 g}+A_{2 g}+\left[\left[E_{u}\right]\right]
$$

(b) $D$-point with accoustic $D_{1}+D_{3}$ and optical $D_{2}+D_{4}$ magnons:

$$
\begin{gathered}
D_{1}^{2}+D_{3}^{2}=D_{2}^{2}+D_{4}^{2}=A_{1 g}+E_{g}+\left[\left[A_{1 g}+E_{g}\right]\right], \\
2 D_{1} \times D_{3}=2 D_{2} \times D_{4}=2\left[\left[A_{2 g}+E_{g}\right]\right], \\
D_{1} \times D_{2}+D_{3} \times D_{4}=A_{1 u}+E_{u}+\left[\left[A_{1 u}+E_{u}\right]\right],
\end{gathered}
$$

$$
D_{1} \times D_{4}+D_{2} \times D_{3}=A_{2 u}+E_{u}+\left[\left[A_{2 u}+E_{u}\right]\right] ;
$$

(c) $A$-point with accoustic $A_{1}$ and optical $A_{1}$ magnons:

$$
\begin{gathered}
{\left[A_{1}^{2}\right]=\left[\left[A_{1 g}+A_{2 g}+2 E_{g}\right]\right]+A_{1 u}+E_{u},} \\
A_{1}^{2}=\left[\left[A_{2 u}+E_{u}\right]\right],
\end{gathered}
$$

for each mode, and

$$
A_{1} \times A_{1}=A_{1 g}+A_{2 g}+2 E_{g}+\left[\left[A_{1 u}+A_{2 u}+2 E_{u}\right]\right]
$$

for the simultaneons excitation of the acoustic and optical mode.

For $D_{3 d}$, the states of allowed electric dipole transition are $E_{u}$ for $a$ - and $b$-polarized light and $A_{2 u}$ for $c$-polarization and the allowed states of Raman scattering are $E_{g}$ and $A_{1 g}$. We have already obtained the eigen-vectors at high-symmetry points [10] and we have also eigen-energies of the magnon at these points [16] as shown in Table 1.

Now we can assign the two-magnon excitation from the experimental results of midinfrared absorption and Raman scattering as well as from the selection rule together with Table 1. First, we can eliminate the contribution from $Z$-point to the midinfrared absorption of two-magnon excitation because $\left[\left[A_{2 u}\right]\right]$ in Eq. (1) and $\left[\left[E_{u}\right]\right]$ in Eq. (2) come from two magnons within the up- or down-spin sublattices. Furthermore Raman scattering with $E_{g}$ symmetry is absent from the contribution at $Z$-point. Second, the dipole-allowed transition with $E_{u}$ symmetry is possible from $D$ - and $A$-points as seen from Eqs. (5),(6) and Eq. (7), respectively. However, the doublet structure of $E_{g}$ Raman scattering is acceptable for $D$-point because the splitting between two acoustic magnons $D_{1} \times D_{3}$ and two optical magnons $D_{2} \times D_{4}$ in Eq. (3) give the doublet structure of $E_{g}$ symmetry with the splitting energy $10 \mathrm{meV}$ in agreement with the observed value. The Raman signal in Eq. (9) comes from one optical and one acoustic magnons and the splitting of two $E_{g}$ modes may be speculated to be smaller than the splitting $1.4 \mathrm{meV}$ between acoustic and optical magnons. Although we cannot eliminate the possibility to assign the doublet Raman signal to the two-magnon excitation at $A$-point, two-magnon excitation from $D$-point looks most probable.

Table 1. Representations and excitation energies of magnons at high-symmetry points.

\begin{tabular}{c|c|c}
\hline \hline Symmetry point & Representaion & Energy, meV \\
\hline \hline ए optical & $E_{u}$ & 97.0 \\
$Z$ acoustic & $Z_{2}$ & 86.8 \\
optical & $Z_{3}$ & 90.9 \\
$D$ acoustic & $D_{1}+D_{3}$ & 92.0 \\
optical & $D_{2}+D_{4}$ & 97.1 \\
$A$ acoustic & $A_{1}$ & 96.6 \\
optical & $A_{1}$ & 98.0 \\
\hline \hline
\end{tabular}

We shall discuss the possibility of this assignment from numerical points. A combination energy of infrared-active $\left(D_{1}+D_{3}\right)$ and $\left(D_{2}+D_{4}\right)$ is $189 \mathrm{meV}(1533$ $\mathrm{cm}^{-1}$ ) while two overtones of Raman-active $D_{1}^{2}+D_{3}^{2}$ and $D_{2}^{2}+D_{4}^{2}$ are $184 \mathrm{meV}\left(1482 \mathrm{~cm}^{-1}\right)$ and $194 \mathrm{meV}$ $\left(1565 \mathrm{~cm}^{-1}\right)$, respectively. The systematic difference between the observed energies and the sum of corresponding energies given in Table 1 is about $30 \mathrm{~cm}^{-1}$ and may be attributed to the binding energy of two magnons [28]. While a combination of acoustic and optical magnons $D_{1} \times D_{4}+D_{2} \times D_{3}$ in Eq. (6) may be excited by both polarized light $\mathbf{E} \perp c$-axis $\left(E_{u}\right)$ and $\mathbf{E} \| c$-axis $\left(A_{2 u}\right)$, the effective dipole moment of this process is by an order of magnitude smaller than that of Eq. (5) [10]. Therefore the midinfrared absorption due to two-magnon excitation should be observed dominantly under $\mathbf{E} \perp c$-axis through $E_{u}$ in Eq. (5). 
We shall show that three-magnon excitation is accessible by both incident beams with the polarization parallel and perpendicular to $c$-axis. The three-magnon excitation may be possible for the combination of an optical magnon with $E_{u}$ symmetry at $\Gamma$-point and two magnons at the opposite BZ edges. We may use $D$-points as the BZ edge point with high symmetry. Then the three-magnon excitations have the following symmetry:

$$
\begin{gathered}
E_{u} \times\left(D_{1}^{2}+D_{3}^{2}\right)=E_{u} \times\left(D_{2}^{2}+D_{4}^{2}\right)= \\
=A_{1 u}+A_{2 u}+2 E_{u}+\left[\left[A_{1 u}+A_{2 u}+2 E_{u}\right]\right], \\
E_{u} \times\left(D_{1} \times D_{2}+D_{3} \times D_{4}\right)= \\
=E_{u} \times\left(D_{1} \times D_{4}+D_{2} \times D_{4}\right)= \\
=A_{1 g}+A_{2 g}+2 E_{g}+\left[\left[A_{1 g}+A_{2 g}+2 E_{g}\right]\right] .
\end{gathered}
$$

As a result, three-magnon excitations $E_{u} \times$ $\times\left(D_{1}^{2}+D_{3}^{2}\right)$ and $E_{u} \times\left(D_{2}^{2}+D_{4}^{2}\right)$ are excited by the incident beam with both polarizations parallel $\left(A_{2 u}\right)$ and perpendicular $\left(E_{u}\right)$ to $c$-axis. The excitation energy which is estimated as a sum of the corresponding magnon energies in Table 1, extends from $281 \mathrm{meV}$ $\left(2266 \mathrm{~cm}^{-1}\right)$ to $291 \mathrm{meV}\left(2349 \mathrm{~cm}^{-1}\right)$. The energy difference of the observed one from these is a little larger than $30 \mathrm{~cm}^{-1}$ (the binding energy of two magnons [28]) and may be attributed to the binding energy of three magnons.

\section{Microscopic model}

The possibility of electric dipole transition due to two- and three-magnon excitation has been confirmed by the group-theoretical consideration and the magnon-dispersion obtained by the neutron diffraction. In the two-magnon excitation, the superexchange interaction between the neighboring antiferromagnetic ions is accompanied with the electric dipole transition within the transition-metal ion or the charge-transfer transition between the empty $3 d$-orbital of the magnetic ion and the $2 p$-orbitals of the surrounding oxygen ion [9]. We use the expression in Ref. 9 to estimate the two-magnon absorption coefficient. The charge transfer excitation gives the absorption coefficient of an order of $10^{4} \mathrm{~cm}^{-1}$ around $2 \mathrm{eV}$. The superexchange interaction is assumed to be of an order of Néel temperature $960 \mathrm{~K}(0.1 \mathrm{eV})$, and we use this value for the off-diagonal exchange matrix element. Then the absorption coefficient for two-magnon excitation is estimated to be of an order of $10^{2} \mathrm{~cm}^{-1}$. This value is reasonable to explain the observed results in Fig. 1. The advantage of direct optical excitation of two magnons in $\alpha-\mathrm{Fe}_{2} \mathrm{O}_{3}$ over $\mathrm{MnF}_{2}$ is speculated to arise from the large charge-transfer matrix element between $\mathrm{O}(2 p)$ and
$\mathrm{Fe}^{3+}(3 d)$ orbitals and its low excitation energy $\Delta E$ of an order of $2 \mathrm{eV}$ for $\alpha-\mathrm{Fe}_{2} \mathrm{O}_{3}$. This large matrix element at the same time results in large absorption coefficient of an order of $10^{4} \mathrm{~cm}^{-1}$ and high Néel temperature $960 \mathrm{~K}$ through the large superexchange integral $2 J$. This is in contrast to Néel temperature $67.3 \mathrm{~K}$ of $\mathrm{MnF}_{2}$.

The dipole moment of two-magnon excitation is described by $\pi(i, j) \mathbf{S}_{i} \cdot \mathbf{S}_{j}$. As to the three-magnon excitation, we first remember that Moriya [15] has derived the so-called Dzyaloshinski-Moriya interaction $\mathbf{d} \cdot\left(\mathbf{S}_{i} \times \mathbf{S}_{j}\right)$ as the (off-diagonal) exchange interaction $-2 J \mathbf{S}_{i} \cdot \mathbf{S}_{j}$ between the ions $i$ and $j$ modified by the (off-diagonal) spin-orbit interaction $\lambda(\mathbf{S} \cdot \mathbf{L})$. The magnitude of $\mathbf{d}$ here may be estimated very roughly as $(\lambda / \Delta E) \times(-2 J)$, where $\Delta E$ is the energy of electronic excitation from the ground $|0\rangle$ to the excited state $|n\rangle$. This suggests that there may also exist an effective dipole moment such as $\pi^{D M}(i, j) \cdot\left(\mathbf{S}_{i} \times \mathbf{S}_{j}\right)$ which may be regarded as derived from the exchange dipole moment $\pi(i, j) \mathbf{S}_{i} \cdot \mathbf{S}_{j}$ (responsible for the two-magnon absorption) perturbed by the spin-orbit interaction. The magnitude of $\boldsymbol{\pi}^{D M}$ may then be given roughly by $(\lambda / \Delta E) \times \boldsymbol{\pi}$. Note here that $\mathbf{S}_{i} \times \mathbf{S}_{j}$ contains, e.g., a term like $\left(S_{i x}-i S_{i y}\right) S_{j z}-S_{i z}\left(S_{j x}-i S_{j y}\right)$ which gives rise to three-magnon excitation.

The expression for the effective dipole moment $\mathbf{P}^{D M}$ of three-magnon excitation is expressed as

$$
\mathbf{P}^{(D M)}=\mathbf{P}_{i^{*} j}^{(D M)}+\underset{j^{*} i}{\boldsymbol{P}^{*}{ }^{(D M)}},
$$

$$
\begin{aligned}
\mathbf{P}_{i^{*} j}^{(D M)} & =\sum_{n}\left(\frac{\left\langle 00\left|\mathcal{H}_{\mathrm{so}}\right| n 0 \backslash\left\langle n 0\left|\mathbf{P}_{i^{*} j}\right| 00\right\rangle\right.}{E_{i 0}-E_{\text {in }}}+\right. \\
+ & \left.\frac{\left\langle 00\left|\mathbf{P}_{i^{*}{ }^{*}}\right| n 0\right\rangle\left\langle n 0\left|\mathcal{H}_{\mathrm{so}}\right| 00\right\rangle}{E_{i 0}-E_{\text {in }}}\right)
\end{aligned}
$$

with the exchange dipole $\mathbf{P}_{i^{*} j}$ defined in Ref. 9. That is,

$$
\begin{gathered}
\mathbf{P}_{i^{*} j}=\sum_{n n^{\prime} m} \pi\left(i ; n^{\prime} \leftarrow n, j m\right) \mathbf{s}_{i}\left(n^{\prime} \leftarrow n\right) \cdot \mathbf{s}_{j m}, \\
\mathbf{s}_{i}\left(n^{\prime} \leftarrow n\right)=\sum_{m m^{\prime}} c_{i n^{\prime} m^{\prime}}^{\dagger} c_{i n m}\left\langle m^{\prime}|\mathbf{s}| m\right\rangle .
\end{gathered}
$$

When we assume that the ion $i$ is excited by the light to the lowest orbital state $|n\rangle=\left|\kappa T_{1 g} v\right\rangle$ from its ground state $|0\rangle=\left|A_{1 g}\right\rangle$, we have the following matrix element:

$$
\left\langle n 0\left|\mathbf{P}_{i^{*} j}\right| 00\right\rangle=\boldsymbol{\Pi}(n 0,00)\left\langle S-1 M_{S}^{0}\left|\boldsymbol{\sigma}_{i}\right| S M_{S}\right\rangle \cdot\left\langle 0\left|\mathbf{S}_{j}\right| 0\right\rangle,
$$




$$
\begin{gathered}
\Pi(n 0,00)=\sum_{n n^{\prime} m} \pi\left(i ; n^{\prime} \leftarrow n, j m\right) \times \\
\times \frac{\left\langle\kappa^{4} T_{1 g} v\left\|\mathbf{s}_{i}\left(n^{\prime} \leftarrow n\right)\right\|^{6} A_{1 g}\right\rangle}{\langle S-1\|\sigma\| S\rangle 2 S} .
\end{gathered}
$$

In terms of operator equivalent for off-diagonal elements $[29,30], \mathcal{H}_{\text {so }}$ may be replaced by $\zeta(0 n) \sigma^{\dagger} \cdot \tau^{\dagger}$.

$$
\begin{gathered}
\left\langle{ }^{6} A_{1 g} M_{s}\left|\mathcal{H}_{\mathrm{so}}\right| \kappa^{4} T_{1 . g} M^{0} v\right\rangle= \\
=\zeta(0 n)\left\langle S M_{s}\left|\sigma^{\dagger}\right| S-1 M_{s}^{0}\right\rangle \cdot\left\langle A_{1 g}\left|\tau^{\dagger}\right| \kappa T_{1 g} v\right\rangle,( \\
\text { where } \zeta(0 n)=\frac{\left\langle{ }^{6} A_{1 g}\left\|V_{\mathrm{so}}\right\| \kappa^{4} T_{1 g}\right\rangle}{\left\langle S\left\|\sigma^{\dagger}\right\| S-1\right\rangle \cdot\left\langle A_{1 g}\left\|\tau^{\dagger}\right\| \kappa T_{1 g}\right\rangle}, \\
\left\langle\kappa T_{1 g}\|\tau\| A_{1 g}\right\rangle=\left\langle A_{1 g}\left\|\tau^{\dagger}\right\| \kappa T_{1 g}\right\rangle=i \sqrt{3}, \\
\langle S-1\|\sigma\| S\rangle=-\left\langle S\left\|\sigma^{\dagger}\right\| S-1\right\rangle=\sqrt{(2 S+1) / 2}
\end{gathered}
$$

The reduced matrix element of the spin-orbit interaction operator $V_{\text {so }}$ is found in Ref. 31:

$$
\left\langle{ }^{6} A_{1 g}\left\|V_{\mathrm{so}}\right\| \mathrm{\kappa}^{4} T_{1 g}\right\rangle=6 i \zeta
$$

with $\zeta$, the spin-orbit coupling parameter for the $3 d$-orbital. The operator $\tau$ is defined as

$$
\begin{aligned}
& \tau_{v}\left|A_{1 g}\right\rangle=i\left|\kappa T_{1 g} v\right\rangle, \\
& \left\langle A_{1 g}\right| \tau_{v}^{\dagger}=-i\left\langle\kappa T_{1 g} v\right|,
\end{aligned}
$$

for $v=\alpha, \beta, \gamma$, and

$\left\langle n\left|\mathcal{H}_{\mathrm{so}}\right| 0\right\rangle=\zeta(0 n)^{*}\left\langle S-1 M_{s}^{0}|\sigma| S M_{s}\right\rangle \cdot\left\langle\kappa T_{1 g} v|\tau| A_{1 g}\right\rangle$,

where $S=5 / 2$.

We now have

$$
\begin{gathered}
\mathbf{P}_{i^{*} j}^{(D M)}=\sum_{n}\left[\frac{\left\langle 00\left|\zeta(0 n) \sigma_{i}^{\dagger} \cdot \tau_{i}^{\dagger}\right| n 0\right\rangle\left\langle n 0\left|\mathbf{P}_{i^{*} j}\right| 00\right\rangle}{E_{i 0}-E_{i n}}+\right. \\
\left.+\frac{\left\langle 00\left|\mathbf{P}_{i^{*} j}\right| n 0\right\rangle\left\langle n 0\left|\zeta(0 n)^{*} \boldsymbol{\sigma}_{i} \cdot \tau_{i}\right| 00\right\rangle}{E_{i 0}-E_{i n}}\right]= \\
=\sum_{n} \zeta(0 n) \sum_{\alpha \beta}\left[\frac{\Pi(n 0,00)}{E_{i 0}-E_{i n}}\left\langle 0\left|\tau_{\alpha}^{\dagger}\right| n\right\rangle \sigma_{i \alpha}^{\dagger} \sigma_{i \beta} S_{j \beta}-\right. \\
\left.-\frac{\Pi(n 0,00)^{*}}{E_{i 0}-E_{i n}}\left\langle 0\left|\tau_{\alpha}^{\dagger}\right| n\right\rangle \sigma_{i \beta}^{\dagger} \sigma_{i \alpha} S_{j \beta}\right]
\end{gathered}
$$

because $\zeta(0 n)^{*}=\zeta(0 n)$.

We then obtain

$$
\begin{aligned}
\mathbf{P}^{(D M)} & =\left[\sum_{n} i \zeta(0 n) \frac{\operatorname{Re} \boldsymbol{\Pi}(n 0,00)}{E_{i 0}-E_{i n}}\left\langle 0\left|\tau_{i}^{\dagger}\right| n\right\rangle-\right. \\
-\sum_{m} i \zeta(0 m) & \left.\frac{\operatorname{Re} \boldsymbol{\Pi}(m 0,00)}{E_{j 0}-E_{j m}}\left\langle 0\left|\tau_{j}^{\dagger}\right| m\right\rangle\right] \cdot \frac{-1}{2 S}\left(\mathbf{S}_{i} \times \mathbf{S}_{j}\right)+ \\
+ & \sum_{n} i \zeta(0 n) \frac{\operatorname{Im} \boldsymbol{\Pi}(n 0,00)}{E_{i 0}-E_{i n}}\left\langle 0\left|\tau_{i}^{\dagger}\right| n\right\rangle \times \\
& \times\left(\frac{1}{3} \mathbf{S}_{j}-\frac{1}{S(2 S-1)} \mathbf{Q}_{i} \cdot \mathbf{S}_{j}\right)+ \\
+ & \sum_{m} i \zeta(0 m) \frac{\operatorname{Im} \boldsymbol{\Pi}(m 0,00)}{E_{j 0}-E_{j m}}\left\langle 0\left|\tau_{j}^{\dagger}\right| m\right\rangle \times \\
& \times\left(\frac{1}{3} \mathbf{S}_{i}-\frac{1}{S(2 S-1)} \mathbf{Q}_{j} \cdot \mathbf{S}_{i}\right)
\end{aligned}
$$

where we have made use of

$$
\begin{gathered}
\sigma_{\alpha}^{\dagger} \sigma_{\beta}=\delta_{\alpha \beta} / 6+\left[S_{\alpha}, S_{\beta}\right] / 4 S-Q_{\alpha \beta} / 2 S(2 S-1),( \\
Q_{\alpha \beta}=\frac{1}{2}\left(S_{\alpha} S_{\beta}+S_{\beta} S_{\alpha}\right)-\delta_{\alpha \beta} \frac{1}{3} S(S+1),
\end{gathered}
$$

and

$$
\left(\mathbf{Q}_{i} \cdot \mathbf{S}_{j}\right)_{v}=\sum_{\beta} Q_{i ; v \beta} S_{j \beta} .
$$

Here we evaluate $\Pi(n 0,00)$ by choosing $\left|\kappa T_{1 g} a_{+1}\right\rangle$ in the trigonal basis [32] as $|n\rangle$, because this is the orbital state excited by $P_{i^{*} j}$ of Eq. (13) in the magnetically ordered phase. Expanding the $3 d$-orbital in the trigonal field in terms of real $\xi, \eta, \zeta$ and $u, v$ orbitals in the $O_{h}$ field [33], we find that $\boldsymbol{\Pi}(n 0,00)$ is complex. As a consequence, both the vector product term $\left(\mathbf{S}_{i} \times \mathbf{S}_{j}\right)$ and the tensor term $\mathbf{Q}_{i} \cdot \mathbf{S}_{j}$ and $\mathbf{Q}_{j} \cdot \mathbf{S}_{i}$ term in Eq. (27) can contribute to the three-magnon excitation. The three-magnon excitation due to $\mathbf{Q}_{i} \cdot \mathbf{S}_{j}$ and $\mathbf{Q}_{j} \cdot \mathbf{S}_{i}$ terms as well as $\mathbf{S}_{i} \times \mathbf{S}_{j}$ term is always accompanied by the single magnon excitation by $\mathbf{S}_{j}$ or $\mathbf{S}_{i}$ with the common coefficient. We have not observed yet a clear evidence of the single magnon excitation around $800 \mathrm{~cm}^{-1}$ because the tail part of large absorption coefficient due to single phonons below $700 \mathrm{~cm}^{-1}$ prevent clear observation of the single magnon as shown in the inset of Fig. 2. The first term in Eq. (27) corresponds exactly to $\pi^{D M}(i, j) \cdot\left(\mathbf{S}_{i} \times \mathbf{S}_{j}\right)$ obtained in Refs. 8 and 10 simply following Moriya's derivation of the DM interaction. These spin operators are rewritten in terms of the magnon creation and annihilation operators through the Bogoliubov transformation. Then we realize that $\mathbf{S}_{i} \times \mathbf{S}_{j}$ term as well as $\mathbf{Q}_{i} \cdot \mathbf{S}_{j}$ and $\mathbf{Q}_{j} \cdot \mathbf{S}_{i}$ terms con- 
tribute to the three magnon excitation [8]. Note also that the coefficients of both spin-product operators in the transition dipole moment in Eq. (27) is naturally real. From this derivation, we can answer a question where the oscillator strength of three-magnon excitation comes from. That is, when one of the two $\mathrm{Fe}^{3+}$ ions participating in two-magnon excitation is perturbed by off-diagonal elements of the spin-orbit interaction, three-magnon midinfrared absorption becomes possible using the effective exchange dipole moment $\pi(i, j)$. The magnitude of three-magnon absorption due to this process is estimated as follows. The spin-orbit coupling parameter $\lambda(0 n)=\zeta(0 n) / 2 S$ is related to $\zeta$ the atomic one by $\lambda(0 n)=-\zeta / S$. When we use $\zeta=410 \mathrm{~cm}^{-1}$ for $\mathrm{Fe}^{2+}$ of magnitude for $\zeta$ and the excitation energy $\Delta E=8000 \mathrm{~cm}^{-1},(\lambda(0 n) / \Delta E)^{2}$ is estimated to be of an order of $10^{-2} \sim 10^{-3}$. Here either one of the $\mathrm{Fe}^{3+}$ ions which contribute to two-magnon excitation may well accompany the third magnon excitation under the additional action of spin-orbit interaction. Taking into account this fact, the ratio of the absorption coefficient $I_{a b s}$ (3 magnons) $/ I_{a b s}$ (2 magnons) is estimated to be of the order of $10^{-2}$ in agreement with the observed results. At the present stage, we cannot judge theoretically which plays the more important role in the three-magnon excitation, Re $\boldsymbol{\Pi}$ or $\operatorname{Im} \boldsymbol{\Pi}$ in Eq. (27).

\section{Conclusion}

We conclude that two- and three-magnon excitation is induced around $1500 \mathrm{~cm}^{-1}$ and $2200 \mathrm{~cm}^{-1}$, respectively, by the electric dipole moment in the antiferromagnetic $\alpha-\mathrm{Fe}_{2} \mathrm{O}_{3}$. Two magnons at $D$-point have been assigned to contribute to the midinfrared absorption and Raman scattering processes from the polarization dependence and the group theoretical consideration.

In some crystals with a center of inversion such as the layered-perovskites, e.g., $\mathrm{La}_{2} \mathrm{CuO}_{4}$, the mid-infrared absorption due to two-magnon excitation through the exchange dipole moment is not allowed but phonon-assisted two-magnon excitation was observed using the oscillator strength of the phonon. However, this is not the case with $\alpha-\mathrm{Fe}_{2} \mathrm{O}_{3}$ whose unit cell contains four $\mathrm{Fe}^{3+}$ ions with, e.g., (1) up-, (2) down-, (3) down- and (4) up- $\mathrm{Fe}^{3+}$ spins on the $c$-axis. Although a center of inversion is located at the mid-point between 2nd and 3rd, as well as between 4th and $1 \mathrm{st} \mathrm{Fe}^{3+}$ ions, two magnons may be excited from the antiferromagnetic pair of 1 st and $2 \mathrm{nd} \mathrm{Fe}^{3+}$ ions, or that of 3rd and 4th $\mathrm{Fe}^{3+}$ ions with no inversion symmetry around the mid-point. Note that these are the first neighbor pairs. Actually, we have more important 3rd and 4th neighbor (antiferromagnetic) pairs of ions with larger coupling [16] if we take neighboring unit cells into account. No inversion symmetry is found for each of these pairs, which allows them to make significant contributions to the direct optical excitation of two magnons in this crystal [10]. On the contrary, we find that many layered perovskite crystals such as $\mathrm{La}_{2} \mathrm{CuO}_{4}$ and $\mathrm{YBa}_{2} \mathrm{CuO}_{6}$ have the center of inversion between the antiferromagnetic pair of ions, so that only phonon-assisted two-magnon excitations are possible.

The polarization dependence and the absorption spectrum itself of the two-magnon excitation do not change so much above and below the Morin temperature as shown in Figs. 1 and 2, where the sublattice magnetization rotates by $\pi / 2$. This may be explained as follows: the magnon dispersion [17] and optical response of two magnons [10] can be well described by Heisenberg model. This is consistent with the fact that the spin anisotropy energy and anisotropic exchange interaction are much smaller than the isotropic exchange energy [13]. As a result, optical response of two- and three-magnon excitation depends only on the relative angle between the neighboring spins but not on the absolute angle of the sublattice magnetization relative to the crystal-axis. This can explain the other mystery why the polarization characteristic of two-magnon excitation does not change at $T_{M}$.

1. M. Imada, A. Fujimori, and Y. Tokura, Rev. Mod. Phys. 70, 1039 (1998).

2. J.D. Perkin, J.M. Graybeal, M.A. Kastner, R.J. Birgeneau, J.P. Falck, and M. Greven, Phys. Rev. Lett. 71, 1621 (1993); J.D. Perkins, R. J. Birgeneau, J.M. Graybeal, M.A. Kastner, and D.S. Kleinberg, Phys. Rev. B58, 9390 (1998).

3. J.D. Perkins, D.S. Kleinberg, M.A. Kastner, and R.J. Birgeneau, Y. Endoh, K. Yamada, and S. Hosoya, Phys. Rev. B52, R9863 (1995).

4. M. Grüminger, D. van der Marel, A. Damascelli, A. Erb T. Nunner, and T. Kopp, Phys. Rev. B62, 12422 (2000).

5. M. Windt, M. Grüminger, T. Nunner, C. Knetter, K.P. Schmidt, G.S. Uhrig, T. Kopp, A. Freimuth, U. Ammerahl, B. Buchner, and A. Revcolevschi, Phys. Rev. Lett. 87, 127002 (2001).

6. H. Suzuura, H. Yasuhara, A. Furusaki, N. Nagaosa, and Y. Tokura, Phys. Rev. Lett. 76, 2579 (1996).

7. J. Lorenzana and G.A. Zawatzky, Phys. Rev. B52, 9576 (1995); Phys. Rev. Lett. 74, 1867 (1995).

8. S. Azuma, M. Sato, Y. Fujimaki, S. Uchida, Y. Tanabe, and E. Hanamura, Phys. Rev. B71, 014429 (2005).

9. Y. Tanabe, T. Moriya, and S. Sugano, Phys. Rev. Lett. 15, 1023 (1965).

10. Y. Tanabe and E. Hanamura, J. Phys. Soc. Jpn. 74, 670 (2005).

11. S.J. Allen, Jr., R. Loudon, and P.L. Richards, Phys. Rev. Lett. 16, 463 (1966).

12. R. Loudon, Adv. Phys. 13, 423 (1964). 
13. T. Moriya, in: Magnetism I, G.T. Rado and H. Suhl (eds.), Academic Press, New York and London (1963), chap 3, and references cited in this article.

14. I. Dzyaloshinski, J. Phys. Chem. Solids 4, 241 (1958).

15. T. Moriya, Phys. Rev. Lett. 4, 228 (1960).

16. E.J. Samuelsen and G. Shirane, Phys. Status Solidi 42, 241 (1970).

17. E.J. Samuelsen, Physica 43, 353 (1969).

18. S. Onari, T. Arai, and K. Kudo, Phys. Rev. B16, 1717 (1977).

19. I.R. Beattie and T.R. Gilson, J. Chem. Soc. A5, 980 (1970).

20. W. Kappus, Z. Physik B21, 325 (1975). Character Table of Z-point in the Table 1 contains errors which should be replaced by the correct one in Ref. 34 .

21. T.R. Hart, S.B. Adams, and H. Temkin, in: Proceedings of the 3rd International Conference on Light Scattering in Solids, M. Balkanski, R.C.C. Leite, and S. P. S. Porto (eds.), Wiley, New York (1976), p. 259.

22. T.P. Martin, R. Merlin, D.R. Huffman, and M. Cardona, Solid State Commun. 22, 565 (1977).

23. M.J. Massey, U. Baier, R. Merlin, and W.H. Weber, Phys. Rev. B41, 7822 (1990), and references cited in this paper.
24. F.J. Morin, Phys. Rev. 78, 819 (1950).

25. R. Loudon, Phys. Rev. 137, A1784 (1965).

26. R. Loudon, Adv. Phys. 17, 243 (1968).

27. I. Inui, Y. Tanabe, and Y. Onodera, Group Theory and its Applications in Physics (Springer Series in Solid State Sciences 78), Second Corrected Printing, Springer-Verlag, Berlin, Heidelberg and New York (1996), chap. 13.

28. R.J. Elliot and M.F. Thorpe, J. Phys. 2, 1630 (1969).

29. K. Shinagawa and Y. Tanabe, J. Phys. Soc. Jpn. 30, 1280 (1971).

30. T. Fujiwara and Y. Tanabe, J. Phys. Soc. Jpn. 32, 912 (1972).

31. Y. Tanabe, Prog. Theor. Phys. Suppl. 14, 17 (1960).

32. See the appendix of K. Eguchi, Y. Tanabe, T. Ogawa, M. Tanaka, Y. Kawabe, and E. Hanamura, J. Opt. Soc. Am. 22, Jan (2005).

33. S. Sugano, Y. Tanabe, and H. Kamimura, Multiplets of Transition-Metal Ions in Crystals, Academic, New York (1970).

34. E.R. Cowley, Can. J. Phys. 47, 1381 (1969). 